\title{
Anticontrol of Chaos for a Class of Delay Difference Equations Based on Heteroclinic Cycles Connecting Repellers
}

\author{
Zongcheng Li \\ School of Science, Shandong Jianzhu University, Jinan, Shandong 250101, China \\ Correspondence should be addressed to Zongcheng Li; chengzi_0905@163.com
}

Received 1 January 2014; Revised 5 March 2014; Accepted 6 March 2014; Published 13 April 2014

Academic Editor: Ryan Loxton

Copyright (C) 2014 Zongcheng Li. This is an open access article distributed under the Creative Commons Attribution License, which permits unrestricted use, distribution, and reproduction in any medium, provided the original work is properly cited.

\begin{abstract}
This paper is concerned with anticontrol of chaos for a class of delay difference equations via the feedback control technique. The controlled system is first reformulated into a high-dimensional discrete dynamical system. Then, a chaotification theorem based on the heteroclinic cycles connecting repellers for maps is established. The controlled system is proved to be chaotic in the sense of both Devaney and Li-Yorke. An illustrative example is provided with computer simulations.
\end{abstract}

\section{Introduction}

Anticontrol of chaos (or called chaotification) is a process that makes a nonchaotic system chaotic or enhances a chaotic system to produce a stronger or different type of chaos. In recent years, it has been found that chaos can actually be useful under some circumstances, for example, in human brain analysis [1,2], heartbeat regulation [3, 4], encryption [5], digital communications [6], and so forth. So, sometimes it is useful and even important to make a system chaotic or create new types of chaos. This has attracted increasing interest in research on chaotification of dynamical systems due to the great potential of chaos in many nontraditional applications.

In the pursuit of chaotifying discrete dynamical systems, a simple yet mathematically rigorous chaotification method was first developed by Chen and Lai [7-9] from a feedback control approach. After that, many chaotification schemes appeared for discrete dynamical systems based on the feedback control approach. The reader is referred to Chen and Shi [10] and Wang and Chen [11] for a survey of chaotification of discrete dynamical systems, as well as some references cited therein.

It is well known that the time delay appears in many realistic systems with feedback in science and engineering. Meanwhile, it has been shown that introducing delays to an undelayed system can be beneficial, especially for chaotic systems. This is the delayed feedback control method, which is widely used in chaos control. For continuous-time control systems, we refer to [12] and the references therein. In [12], the authors developed a unified computational approach for solving optimal state-delay control problems and proved that the approach was very effective for parameter identification and delayed feedback control. For discrete-time control systems, we refer to [13] and the references therein. In [13], the authors obtained the necessary and sufficient conditions for stabilizability of discrete-time systems via delayed feedback control.

To the best of our knowledge, there are few results on chaotification of delay difference equations. Motivated by the delayed feedback control method, we studied the chaotification problem for a class of delay difference equations with at least two fixed points. Since the sawtooth function and the sine function have some favourable properties, some of which are similar, they are often used as controllers; see [10, 11, 1416] and so forth. Particularly, we succeeded in using the sine function as a controller to chaotify linear delay difference equations in [16]. This motivates us to use the sine function as the controller and employ a feedback control approach to study the chaotification problem for a class of delay difference equations. It is proved that the controlled system is chaotic in the sense of both Devaney and Li-Yorke, by applying the result of heteroclinic cycles connecting repellers; see [17] for the result and some references therein.

The rest of the paper is organized as follows. In Section 2, the chaotification problem under investigation is described, 
and some concepts, lemmas, and reformulation of the controlled system are introduced. In Section 3, the chaotification problem is studied and a chaotification scheme is established. An example is provided to illustrate the theoretical result with computer simulations in Section 4. Finally, Section 5 concludes the paper.

\section{Preliminaries}

In this section, we describe the chaotification problem, give a reformulation of the delay difference equation, and introduce some fundamental concepts and lemmas, which will be used in the next section.

2.1. Description of Chaotification Problem. In this paper, we consider chaotification of the following delay difference equation:

$$
x(n+1)=f(x(n-k), x(n)), \quad n \geq 0,
$$

where $k \geq 1$ is a fixed integer and $f: D \subset \mathbf{R}^{2} \rightarrow \mathbf{R}$ is a map. Equation (1) can be viewed as a discrete analogue of many one-dimensional delay differential equations by using the forward Euler scheme, such as the well-known Mackey-Glass equation

$$
\dot{x}(t)=-\mu x(t)+g(x(t-\tau)),
$$

where $\mu>0, \tau>0$ is the delay, and $g$ is a one-dimensional nonlinear function. Equation (2) is a prototype for a retarded functional differential equation which has many applications in sciences. Special cases of (2) or its discretization have been studied by many authors; for instance, see [18-22] and the references therein.

From the above discussion, we see that the delay difference equation (1) is indeed very general. There exist many papers which are concerned with the existence of chaotic behavior for special forms of functions $f$. For example, see $[21,22]$ and references therein. However, (1) cannot be chaotic for a more general class of functions $f$. The object of this paper is to design a simple control input sequence $\{v(n)\}$ such that the output of the controlled system

$$
x(n+1)=f(x(n-k), x(n))+v(n), \quad n \geq 0,
$$

exhibits chaos in the sense of both Devaney and Li-Yorke for a more general class of functions $f$. The controller to be designed in this paper is in the form of

$$
v(n)=\alpha \sin (\beta x(n-k)),
$$

where $\alpha$ and $\beta$ are two undetermined positive parameters.

For convenience, define $g(x, y):=f(x, y)+\alpha \sin (\beta x)$ throughout the rest of the paper. Let $f_{x}(x, y)$ and $f_{y}(x, y)$ denote the first-order partial derivatives of $f$ with respect to the first and the second variables at the point $(x, y)$, respectively. In the following, by $B_{r}(z)$ and $\bar{B}_{r}(z)$ denote the open and closed balls of radius $r$ centered at $z$.
2.2. Reformulation. Here, we reformulate (1) and (3) into two special high-dimensional discrete dynamical systems. The following transform method is used by many researchers; for example, see $[16,21]$ and some references therein.

By setting

$$
u_{j}(n):=x(n+j-k-1), \quad 1 \leq j \leq k+1, n \geq 0,
$$

equation (1) and the controlled system (3) with controller (4) can be written as the following $k+1$-dimensional discrete systems on $\mathbf{R}^{k+1}$ :

$$
\begin{aligned}
& u(n+1)=F(u(n)), \\
& u(n+1)=G(u(n)),
\end{aligned}
$$

respectively, where $u=\left(u_{1}, u_{2}, \ldots, u_{k+1}\right)^{T} \in \mathbf{R}^{k+1}$, and the maps $F, G: \mathbf{R}^{k+1} \rightarrow \mathbf{R}^{k+1}$ are given by

$$
\begin{gathered}
F\left(\begin{array}{c}
u_{1}(n) \\
u_{2}(n) \\
\vdots \\
u_{k}(n) \\
u_{k+1}(n)
\end{array}\right)=\left(\begin{array}{c}
u_{2}(n) \\
u_{3}(n) \\
\vdots \\
u_{k+1}(n) \\
f\left(u_{1}(n), u_{k+1}(n)\right)
\end{array}\right) \\
G\left(\begin{array}{c}
u_{1}(n) \\
u_{2}(n) \\
\vdots \\
u_{k}(n) \\
u_{k+1}(n)
\end{array}\right) \\
=\left(\begin{array}{c}
u_{2}(n) \\
u_{3}(n) \\
\vdots \\
u_{k+1}(n) \\
(n))+\alpha \sin \left(\beta u_{1}(n)\right)
\end{array}\right) .
\end{gathered}
$$

The map $G$ is called the map induced by $g$. System (7) is called the system induced by (3) in the Euclidean space $\mathbf{R}^{k+1}$. It is evident that a solution $\{x(n-k), \ldots, x(n)\}_{n=1}^{\infty}$ of (3) with an initial condition $\{x(-k), \ldots, x(0)\}$ corresponds to a solution $\{u(n)\}_{n=1}^{\infty}$ of system (7) with an initial condition $u(0)=\left(u_{1}(0), \ldots, u_{k+1}(0)\right)^{T} \in \mathbf{R}^{k+1}$. We say that the solution $\{u(n)\}_{n=1}^{\infty}$ of system (7) is induced by the solution $\{x(n-k)$, $\ldots, x(n)\}_{n=1}^{\infty}$ of (3). Therefore, we can investigate the dynamical behavior of system (3) by investigating that of its induced system (7) in $\mathbf{R}^{k+1}$. There is the same conclusion between systems (6) and (1). The idea in the above definitions is motivated by [21], where the authors say that the induced system and the original system are equivalent.

2.3. Some Basic Concepts and Lemmas. Since Li and Yorke [23] first introduced a precise mathematical definition of chaos, there have been several different definitions of chaos, 
some stronger and some weaker, depending on the requirements in different problems; see [24-27] and so forth. For convenience, we list two definitions of chaos in the sense of Li-Yorke and Devaney, which are used in this paper.

Definition 1. Let $(X, d)$ be a metric space, $F: X \rightarrow X$ a map, and $S$ a set of $X$ with at least two distinct points. Then $S$ is called a scrambled set of $F$ if for any two distinct points $x, y \in$ $S$,

(i) $\liminf _{n \rightarrow \infty} d\left(F^{n}(x), F^{n}(y)\right)=0$;

(ii) $\limsup _{n \rightarrow \infty} d\left(F^{n}(x), F^{n}(y)\right)>0$.

The map $F$ is said to be chaotic in the sense of Li-Yorke if there exists an uncountable scrambled set $S$ of $F$.

Remark 2. There are three conditions in the original characterization of chaos in Li-Yorke's theorem [23]. Besides conditions (i) and (ii), the third one is that, for all $x \in S$ and for all periodic points $p$ of $F$,

$$
\limsup _{n \rightarrow \infty} d\left(F^{n}(x), F^{n}(p)\right)>0 \text {. }
$$

But conditions (i) and (ii) together imply that the scrambled set $S$ contains at most one point $x$ that does not satisfy the above condition. Hence, the third condition is not essential and can be removed.

Definition 3 (see [24]). Let $(X, d)$ be a metric space. A map $F: V \subset X \rightarrow V$ is said to be chaotic on $V$ in the sense of Devaney if

(i) the set of the periodic points of $F$ is dense in $V$;

(ii) $F$ is topologically transitive in $V$;

(iii) $F$ has sensitive dependence on initial conditions in $V$.

Remark 4. By the result of Banks et al. [28], conditions (i) and (ii) together imply condition (iii) if $F$ is continuous in $V$. Consequently, condition (iii) is redundant in the above definition if $F$ is continuous in $V$. It has been proved in [29] that, under some conditions, chaos in the sense of Devaney is stronger than that in the sense of Li-Yorke.

For convenience, some definitions of relevant concepts given in [30] are listed below.

Definition 5 (see [30, Definitions 2.1 and 2.4]). Let $(X, d)$ be a metric space and $F: X \rightarrow X$ a map. A point $z \in X$ is called an expanding fixed point (or a repeller) of $F$ in $\bar{B}_{r}(z)$ for some constant $r>0$, if $F(z)=z$ and there exists a constant $\lambda>1$ such that

$$
d(F(x), F(y)) \geq \lambda d(x, y) \quad \forall x, y \in \bar{B}_{r}(z) .
$$

The constant $\lambda$ is called an expanding coefficient of $F$ in $\bar{B}_{r}(z)$. Furthermore, $z$ is called a regular expanding fixed point of $F$ in $\bar{B}_{r}(z)$ if $z$ is an interior point of $F\left(B_{r}(z)\right)$. Otherwise, $z$ is called a singular expanding fixed point of $F$ in $\bar{B}_{r}(z)$.
Now, we introduce some relative concepts for system (3), which are motivated by [15, Definitions 5.1 and 5.2]. There are identical concepts for system (1).

Definition 6. Consider the following.

(i) A point $x \in \mathbf{R}^{k+1}$ is called an $m$-periodic point of system (3) if $x \in \mathbf{R}^{k+1}$ is an $m$-periodic point of its induced system (7); that is, $F^{m}(x)=x, F^{j}(x) \neq x$, and $1 \leq j \leq m-1$. In the special case of $m=1, x$ is called a fixed point or a steady state of system (3).

(ii) The concepts of density of periodic points, topological transitivity, sensitive dependence on initial conditions, and the invariant set for system (3) are defined similarly to those for its induced system (7) in $\mathbf{R}^{k+1}$.

(iii) System (3) is said to be chaotic in the sense of Devaney (or Li-Yorke) on $V \subset \mathbf{R}^{k+1}$ if its induced system (7) is chaotic in the sense of Devaney (or Li-Yorke) on $V \subset \mathbf{R}^{k+1}$

The following two lemmas will be used in the next section.

Lemma 7. Assume that the map $f$ in (1) is continuously differentiable in a neighborhood of $(0,0)$ with $f(0,0)=0$ and satisfies

$$
\left|f_{x}(0,0)\right|-\left|f_{y}(0,0)\right|>1
$$

then the fixed point $O:=(0, \ldots, 0)^{T} \in \mathbf{R}^{k+1}$ of system (6) is a regular expanding fixed point in some norm in $\mathbf{R}^{k+1}$.

Proof. It follows from $f(0,0)=0$ that $O$ is a fixed point of system (6). Since $f$ is continuously differentiable in a neighborhood of $(0,0), F$ is continuously differentiable in some neighborhood of $O$. The Jacobian matrix of map $F$ in system (6) at $O$ is

$$
D F(O)=\left(\begin{array}{ccccc}
0 & 1 & 0 & \cdots & 0 \\
0 & 0 & 1 & \cdots & 0 \\
\cdots & \cdots & \cdots & \cdots & \cdots \\
0 & 0 & 0 & \cdots & 1 \\
f_{x}(0,0) & 0 & 0 & \cdots & f_{y}(0,0)
\end{array}\right)_{(k+1) \times(k+1)}
$$

and its characteristic equation is

$$
\lambda^{k+1}-f_{y}(0,0) \lambda^{k}-f_{x}(0,0)=0 .
$$

From $\left|f_{x}(0,0)\right|-\left|f_{y}(0,0)\right|>1$, we can show that all the eigenvalues of $D F(O)$ have absolute values larger than 1 . Otherwise, suppose that there exists an eigenvalue $\lambda_{0}$ of $D F(O)$ with $\left|\lambda_{0}\right| \leq 1$; then we get the following inequality:

$$
\begin{aligned}
1+\left|f_{y}(0,0)\right| & \geq\left|\lambda_{0}^{k+1}-f_{y}(0,0) \lambda_{0}^{k}\right| \\
& =\left|f_{x}(0,0)\right|>1+\left|f_{y}(0,0)\right|,
\end{aligned}
$$

which is a contradiction. Then it follows from [31, Theorem 4.3] that there exist a positive constant $r^{*}$ and a norm $\|\cdot\|^{*}$ in 
$\mathbf{R}^{k+1}$ such that $F$ is continuously differentiable in $\bar{B}_{r^{*}}(O)$ and $O$ is an expanding fixed point of $F$ in $\bar{B}_{r^{*}}(O)$ in the norm $\|\cdot\|^{*}$; that is,

$$
\|F(x)-F(y)\|^{*} \geq \mu\|x-y\|^{*}, \quad \forall x, y \in \bar{B}_{r^{*}}(O),
$$

where $\mu>1$ is an expanding coefficient of $F$ in $\bar{B}_{r^{*}}(O)$. Further, it follows from [30, Lemma 2.2] that $O$ is a regular expanding fixed point of system (6) in the norm $\|\cdot\|^{*}$ in $\mathbf{R}^{k+1}$. This completes the proof.

Since the result in the following lemma is related to the one-sided symbolic dynamical system $\left(\sum_{2}^{+}, \sigma\right)$, we briefly recall some results of it for convenience. Let

$$
\sum_{2}^{+}:=\left\{s=\left(s_{0}, s_{1}, s_{2}, \ldots\right): s_{j}=0 \text { or } 1\right\}
$$

with the distance

$$
\rho(s, t):=\sum_{i=0}^{\infty} \frac{\left|s_{i}-t_{i}\right|}{2^{i}}
$$

where $s=\left(s_{0}, s_{1}, s_{2}, \ldots\right)$ and $t=\left(t_{0}, t_{1}, t_{2}, \ldots\right)$. Then $\left(\sum_{2}^{+}, \rho\right)$ is a complete metric space and a Cantor set. The shift map $\sigma: \sum_{2}^{+} \rightarrow \sum_{2}^{+}$defined by $\sigma\left(\left(s_{0}, s_{1}, s_{2}, \ldots\right)\right)=$ $\left(s_{1}, s_{2}, \ldots\right)$ is continuous. The dynamical system defined by $\sigma$ is called a one-sided symbolic dynamical system. It has plentiful dynamical behaviors; we refer to $[24,26]$ for details. Particularly, it is chaotic in the sense of both Devaney and LiYorke and has a positive topological entropy.

Lemma 8 (see [17, Theorem 4.3 and Corollary 4.2]). Let a map $f: \mathbf{R}^{n} \rightarrow \mathbf{R}^{n}$ have $k(\geq 2)$ different fixed points $z_{1}, \ldots$, $z_{k} \in \mathbf{R}^{n}$. Assume that

(i) for each $i, 1 \leq i \leq k, z_{i}$ is an expanding fixed point of $f$ in some norm $\|\cdot\|_{i}$;

(ii) $f$ has a $k$-heteroclinic cycle connecting fixed points $z_{1}, \ldots, z_{k}$ and is continuously differentiable in some neighborhood of each point $x_{0}$ on the cycle satisfying $\operatorname{det} D f\left(x_{0}\right) \neq 0$.

Then for each $i, 1 \leq i \leq k$, and for each neighborhood $U_{i}$ of $z_{i}$, there exist a positive integer $n_{i}$ and a Cantor set $\Lambda_{i} \subset U_{i}$ such that $f^{n_{i}}: \Lambda_{i} \rightarrow \Lambda_{i}$ is topologically conjugate to the one-sided symbolic dynamical system $\sigma: \sum_{2}^{+} \rightarrow \sum_{2}^{+}$. Consequently, there exists a compact and perfect invariant set $D_{i}=\bigcup_{j=0}^{n_{i}-1} f^{j}\left(\Lambda_{i}\right) \subset$ $\mathbf{R}^{n}$, containing the Cantor set $\Lambda_{i}$, such that $f$ is chaotic on $D_{i}$ in the sense of Devaney as well as in the sense of Li-Yorke.

Remark 9. Under the conditions in Lemma 8, there exists a positive integer $p$, such that $f^{p}$ has a regular and nondegenerate $k$-heteroclinic cycle connecting repellers $z_{1}, \ldots, z_{k}$ in the Euclidean norm $\|\cdot\|$. Therefore, Lemma 8 can be briefly stated as the following: "a regular and nondegenerate heteroclinic cycle connecting repellers in $\mathbf{R}^{n}$ implies chaos in the sense of both Devaney and Li-Yorke." We refer to [17] for details.

\section{A Chaotification Scheme}

In this section, a chaotification scheme for the controlled system (3) with controller (4) is established for the case that the original system (1) has at least two fixed points. Here, we only need that the map $f$ of the original system is continuously differentiable in a region containing two fixed points. In the case that the fixed points are more than two, if two of them satisfy conditions in the following theorem, then we can choose the two fixed points to establish a chaotification scheme by using Lemma 8 for $k=2$. If none of the two fixed points is the origin $O$, then we can choose a transformation of coordinates such that one fixed point becomes the origin $O$ in a new coordinate system. Therefore, without loss of generality, we only discuss the case that the original system $(1)$ has two fixed points $O=(0, \ldots, 0)^{T}$ and $P:=\left(x^{*}, \ldots, x^{*}\right)^{T}$ in $\mathbf{R}^{k+1}$.

Theorem 10. Consider the controlled system (3) with controller (4). Assume that

(i) $f$ is continuously differentiable in $[-r, r]^{2}$ for some $r>$ 0 with $f(0,0)=0$, which implies that there exist positive constants $M$ and $L$ such that for any $(x, y) \in$ $[-r, r]^{2}$

$$
|f(x, y)| \leq M, \quad\left|f_{x}(x, y)\right| \leq L, \quad\left|f_{y}(x, y)\right| \leq L ;
$$

(ii) there exists a point $x^{*} \in(-r, r)$ with $x^{*} \neq 0$ such that $f\left(x^{*}, x^{*}\right)=x^{*}$.

Then there exist two positive constants $\alpha_{0}$ and $\beta_{0}$ satisfying

$$
\alpha_{0}>2\left(M+\left|x^{*}\right|\right), \quad \beta_{0}=\frac{2 m_{0} \pi}{\left|x^{*}\right|}>\frac{1+2 L}{\alpha_{0}},
$$

where $m_{0}$ is some positive integer, such that, for any $\alpha>\alpha_{0}$ and $\beta=\beta_{0}$, the controlled system (3) with controller (4) is chaotic in the sense of both Devaney and Li-Yorke.

Proof. In order to prove that system (3) with controller (4) is chaotic in the sense of both Devaney and Li-Yorke, we only need to prove that its reformulated system (7) is chaotic in the sense of both Devaney and Li-Yorke. Lemma 8 is used to prove this theorem. Thus it suffices to show that all the assumptions in Lemma 8 hold for $k=2$.

For convenience, let $\alpha>\alpha_{0}$ and $\beta=2 m \pi /\left|x^{*}\right|>(1+$ $2 L) / \alpha_{0}$ throughout the proof, where $m$ is an undetermined integer.

By assumption (i), we find that $g$ is continuously differentiable in $[-r, r]^{2}$ and $G$ is continuously differentiable in $[-r, r]^{k+1}$. It is clear that $g(0,0)=0$ and $g\left(x^{*}, x^{*}\right)=x^{*}$. It can also easily be proved that $O$ and $P=\left(x^{*}, \ldots, x^{*}\right)^{T} \in \mathbf{R}^{k+1}$ are two fixed points of $G$. From the last two relations of (18), it follows that

$$
\begin{aligned}
\left|g_{x}(0,0)\right| & =\left|f_{x}(0,0)+\alpha \beta\right| \\
& \geq \alpha \beta-\left|f_{x}(0,0)\right| \geq \alpha \beta-L \\
& >1+L \geq 1+\left|f_{y}(0,0)\right|=1+\left|g_{y}(0,0)\right| .
\end{aligned}
$$


Similarly, it can also be shown that

$$
\left|g_{x}\left(x^{*}, x^{*}\right)\right|=\left|f_{x}\left(x^{*}, x^{*}\right)+\alpha \beta\right|>1+\left|g_{y}\left(x^{*}, x^{*}\right)\right| \text {. }
$$

Then, it follows from Lemma 7 that $O$ and $P$ are two regular expanding fixed points of system (7). That is, there exist two positive constants $r_{1}<r, r_{2}<r$ and two norms $\|\cdot\|_{1},\|\cdot\|_{2}$ in $\mathbf{R}^{k+1}$ such that $O$ and $P$ are two regular expanding fixed points of $G$ in $\bar{B}_{r_{1}}(O)$ in norm $\|\cdot\|_{1}$ and in $\bar{B}_{r_{2}}(P)$ in norm $\|\cdot\|_{2}$, respectively. For convenience, we can choose $r_{1}$ and $r_{2}$ to be very small such that $B_{r_{1}}(O) \cap B_{r_{2}}(P)=\emptyset$.

Next, we need to show that $G$ has a 2-heteroclinic cycle connecting fixed points $O$ and $P$. There exist small intervals $U_{1}$ containing 0 and $U_{2}$ containing $x^{*}$ such that $U_{1} \times U_{1} \times$ $\cdots \times U_{1} \subset B_{r_{1}}(O)$ and $U_{2} \times U_{2} \times \cdots \times U_{2} \subset B_{r_{2}}(O)$. Consider the following equation:

$$
h_{1}(x):=f\left(x, x^{*}\right)+\alpha \sin (\beta x)-x^{*}=0 .
$$

Obviously, $h_{1}$ is continuously differentiable in $[-r, r]$. It is easy to see from (18) that

$$
\begin{gathered}
h_{1}\left(\frac{\left|x^{*}\right|}{4 m}\right)=f\left(\frac{\left|x^{*}\right|}{4 m}, x^{*}\right)+\alpha-x^{*} \geq \alpha-M-\left|x^{*}\right|>0, \\
h_{1}\left(\frac{3\left|x^{*}\right|}{4 m}\right)=f\left(\frac{3\left|x^{*}\right|}{4 m}, x^{*}\right)-\alpha-x^{*} \leq M+\left|x^{*}\right|-\alpha<0,
\end{gathered}
$$

which implies that there exists a point $x_{1}$ with $\left|x^{*}\right| / 4 m<$ $x_{1}<3\left|x^{*}\right| / 4 m$ such that $h_{1}\left(x_{1}\right)=0$ by the continuity of $h_{1}$. Consider the following two equations:

$$
\begin{aligned}
& h_{2}(x):=f\left(x, x_{1}\right)+\alpha \sin (\beta x)-x^{*}=0, \\
& h_{3}(x):=f(x, 0)+\alpha \sin (\beta x)=0 .
\end{aligned}
$$

With a similar method, we can also show that there exist two points $x_{2}$ with $\left|x^{*}\right| / 4 m<x_{2}<3\left|x^{*}\right| / 4 m$ and $x_{3}$ with $\left|x^{*}\right|+\left|x^{*}\right| / 4 m<x_{3}<\left|x^{*}\right|+3\left|x^{*}\right| / 4 m$ such that $h_{2}\left(x_{2}\right)=0$ and $h_{3}\left(x_{3}\right)=0$. Similarly, the following equation

$$
h_{4}(x):=f\left(x, x_{3}\right)+\alpha \sin (\beta x)=0
$$

also has a solution $x_{4}$ with $\left|x^{*}\right|+\left|x^{*}\right| / 4 m<x_{4}<\left|x^{*}\right|+$ $3\left|x^{*}\right| / 4 m$ such that $h_{4}\left(x_{4}\right)=0$. We can choose a large positive integer $m_{0}$ such that for any $m \geq m_{0}$ the points $x_{1}, x_{2}$ are in $U_{1}$ and $x_{3}, x_{4}$ are in $U_{2}$.

Take $O_{0}=\left(x_{2}, x_{1}, \ldots, x_{1}\right)^{T} \in \mathbf{R}^{k+1}$. It is clear that $O_{0} \in$ $B_{r_{1}}(O)$ and $O_{0} \neq O$. Set $O_{j}=G\left(O_{j-1}\right)$ for $1 \leq j \leq k$. We can easily show that $O_{j}=(x_{1}, \ldots, x_{1}, \underbrace{x^{*}, \ldots, x^{*}}_{j})^{T} \notin B_{r_{1}}(O)$ for $1 \leq j \leq k$, and

$$
G^{k+1}\left(O_{0}\right)=P
$$

Take $P_{0}=\left(x_{4}, x_{3}, \ldots, x_{3}\right)^{T} \in \mathbf{R}^{k+1}$. It is also clear that $P_{0} \in B_{r_{2}}(P)$ and $P_{0} \neq P$. Set $P_{j}=G\left(P_{j-1}\right)$ for $1 \leq j \leq k$. It can also easily be shown that $P_{j}=(x_{3}, \ldots, x_{3}, \underbrace{0, \ldots, 0}_{j})^{T} \notin B_{r_{2}}(P)$ for $1 \leq j \leq k$, and

$$
G^{k+1}\left(P_{0}\right)=O
$$

Therefore, $G$ has a 2-heteroclinic cycle connecting repellers $O$ and $P$.

Finally, we will show that

$$
\operatorname{det} D G\left(O_{j}\right) \neq 0, \quad \operatorname{det} D G\left(P_{j}\right) \neq 0, \quad \text { for } 0 \leq j \leq k .
$$

We use the method of proof by contradiction to prove it. For simplicity, we only prove that condition (28) holds for $\mathrm{O}_{0}$. Suppose that det $D G\left(O_{0}\right)=0$. A direct calculation shows that, for any $u=\left(u_{1}, \ldots, u_{k+1}\right)^{T} \in \mathbf{R}^{k+1}$,

$$
\operatorname{det} D G(u)=(-1)^{k}\left[f_{x}\left(u_{1}, u_{k+1}\right)+\alpha \beta \cos \left(\beta u_{1}\right)\right] \text {. }
$$

Then it follows from (29) that

$$
f_{x}\left(x_{2}, x_{1}\right)+\alpha \beta \cos \left(\beta x_{2}\right)=0 .
$$

Hence, $\cos \left(\beta x_{2}\right)=-(1 / \alpha \beta) f_{x}\left(x_{2}, x_{1}\right)$. On the other hand, it follows from $h_{2}\left(x_{2}\right)=0$ that $\sin \left(\beta x_{2}\right)=(1 / \alpha)\left[x^{*}-f\left(x_{2}, x_{1}\right)\right]$. Then, we get the following contradiction:

$$
\begin{aligned}
1 & =\frac{\left[f_{x}\left(x_{2}, x_{1}\right)\right]^{2}}{\alpha^{2} \beta^{2}}+\frac{\left[x^{*}-f\left(x_{2}, x_{1}\right)\right]^{2}}{\alpha^{2}} \\
& <\frac{L^{2}}{(1+2 L)^{2}}+\frac{\left(M+\left|x^{*}\right|\right)^{2}}{4\left(M+\left|x^{*}\right|\right)^{2}}<\frac{1}{2} .
\end{aligned}
$$

Therefore, det $D G\left(O_{0}\right) \neq 0$. Similarly, we can prove that condition (28) holds for $0 \leq j \leq k$.

Therefore, all the assumptions in Lemma 8 are satisfied for $k=2$. It follows from Lemma 8 that, for any $\alpha>\alpha_{0}$ and $\beta=\beta_{0}$, there exists regular and nondegenerate 2 -heteroclinic cycle repellers $O$ and $P$. Consequently, system (7) and thus system (3) with controller (4) are chaotic in the sense of both Devaney and Li-Yorke. This completes the proof.

Remark 11. From the proof of Theorem 10, we see that there exists some positive integer $m_{0}$ such that, for any $\alpha>\alpha_{0}, \beta_{0}=$ $2 m_{0} \pi /\left|x^{*}\right|>(1+2 L) / \alpha_{0}$, system (7) is chaotic in the sense of both Devaney and Li-Yorke. However, it is very difficult to determine the particular integer $m_{0}$ since the expanding area of a fixed point is not easy to obtain, and there are few methods to determine the concrete expanding area of a fixed point in the existing literatures. In practical problems, we can take the positive integer $m_{0}$ large enough such that controller (4) can be used to chaotify system (1).

Remark 12. There are many delay discrete dynamical systems which have more than two fixed points. As all or some of the fixed points satisfy assumptions in Theorem 10, which are not very strict conditions, we can choose two of them and use the chaotification scheme of this paper to chaotify these systems. Since the result of Theorem 10 follows from the result 
of Lemma 8, there will be many chaotic invariant sets when using the chaotification scheme to chaotify these systems. Therefore, the chaotic behaviors induced by a heteroclinic cycle connecting repellers seemed to be more complex than those induced by a single snap-back repeller. The difference between them will be our future research.

\section{An Example}

In the last section, we present an example of chaotification for the delay difference equation (1) with computer simulations. The map $f$ in (1) is taken as follows:

$$
f(x, y)=2 \sin \left[\frac{\pi}{12}(x+y)\right] .
$$

It is obvious that $f$ is continuously differentiable on $\mathbf{R}^{2}$ and satisfies condition (18); that is, for any $(x, y) \in \mathbf{R}^{2}$,

$$
|f(x, y)| \leq 2, \quad\left|f_{x}(x, y)\right| \leq \frac{\pi}{6}, \quad\left|f_{y}(x, y)\right| \leq \frac{\pi}{6},
$$

where $M=2$ and $L=\pi / 6$ in condition (18). It is also clear that $f(0,0)=0$ and there exists a point $x^{*}=1$ such that $f(1,1)=1$. Therefore, all the assumptions in Theorem 10 are satisfied. It follows from Theorem 10 that there exist two positive constants

$$
\begin{aligned}
& \alpha_{0}>2\left(M+\left|x^{*}\right|\right)=6, \\
& \beta_{0}=2 m_{0} \pi=\frac{2 m_{0} \pi}{\left|x^{*}\right|}>\frac{3+\pi}{18}>\frac{1+2 L}{\alpha_{0}},
\end{aligned}
$$

where $m_{0}$ is some positive integer, such that, for any $\alpha>\alpha_{0}$ and $\beta=\beta_{0}$, the controlled system (3) with controller (4) is chaotic in the sense of both Devaney and Li-Yorke.

In fact, it is obvious that the solutions of the uncontrolled system (6) are bounded in $[-2,2]^{2}$ for $k=1$. There are three fixed points for the uncontrolled system (6); that is, $O=$ $(0,0)^{T}, P=(1,1)^{T}, Q=(-1,-1)^{T}$. One can easily verify that $O$ is an unstable fixed point and that $P$ and $Q$ are two stable fixed points. When we take an initial condition $u(0)=$ $(0.01,0.01)^{T}$, the solution $u(n)$ of the uncontrolled system (6) should tend to the stable fixed point $P$ when $n$ tends to infinity. This conforms to Figure 1 , where the curve tends from $(0.01,0.01)^{T}$ to the stable fixed point $(1,1)^{T}$ and $n$ is taken from 0 to 20000 for simulation. There is a similar conclusion for the case $k=2$. For simplicity, we omit it.

In order to help better visualize the theoretical result of Theorem 10, we take $k=1,2, m_{0}=50$ and

$$
\alpha=100, \quad \beta=100 \pi,
$$

for computer simulations. Both of them satisfy the above conditions. Consequently, the controlled system (7) should be chaotic in the sense of both Devaney and Li-Yorke. It is obvious that the solutions of controlled system (7) are bounded in $[-102,102]^{2}$ and $[-102,102]^{3}$ for $k=1$ and $k=2$, respectively. The simulated results in Figures 2 and 4 show that

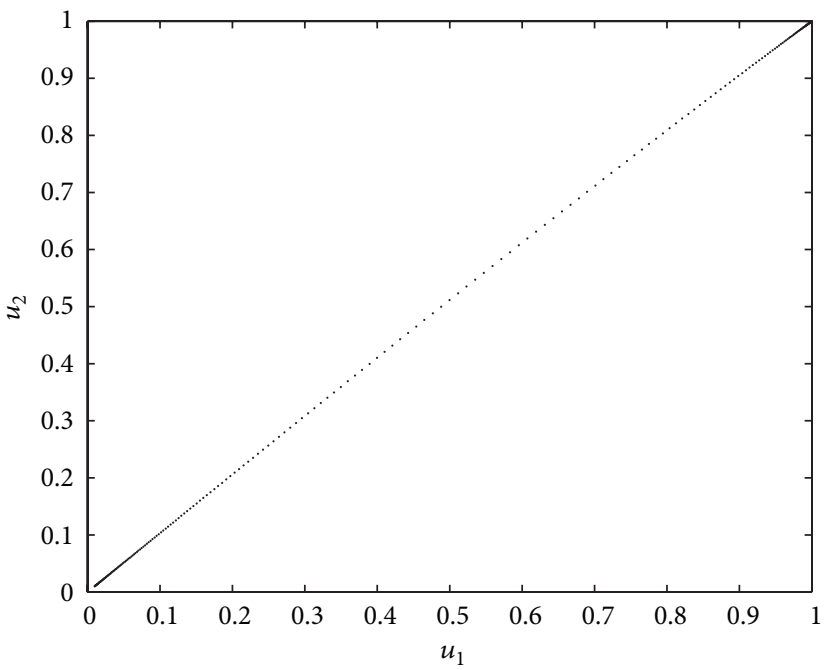

Figure 1: 2D computer simulation result shows simple dynamical behaviors of the uncontrolled system $(6)$ in the $\left(u_{1}, u_{2}\right)$ space for $k=$ 1 , and $n$ from 0 to 20000, with the initial condition $u(0)=(0.01$, $0.01)^{T}$.

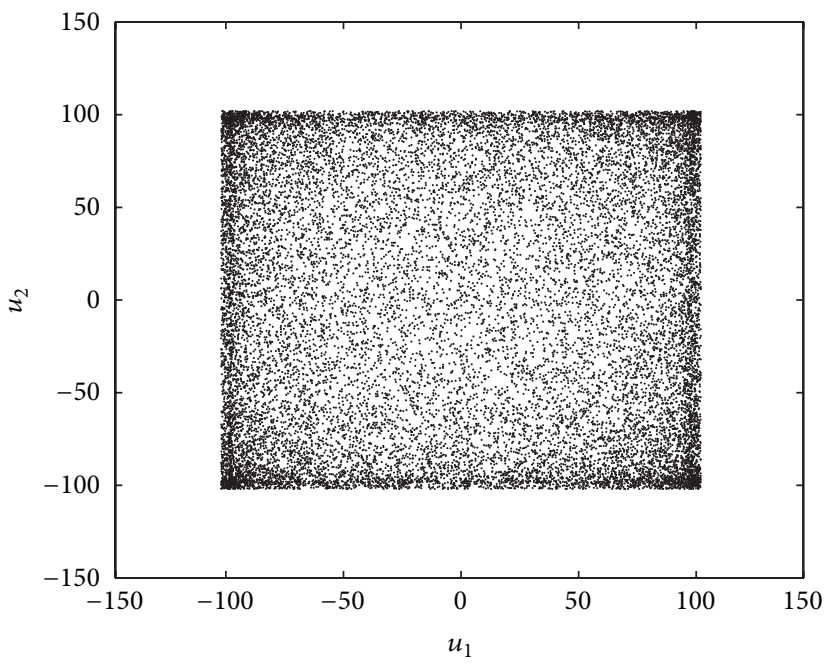

FIGURE 2: 2D computer simulation result shows complex dynamical behaviors of the controlled system (7) in the $\left(u_{1}, u_{2}\right)$ space for $\alpha=$ $100, \beta=100 \pi, k=1$, and $n$ from 0 to 20000 , with the initial condition $u(0)=(0.01,0.01)^{T}$.

the controlled system (7) indeed has complex behaviors, where $n$ is taken from 0 to 20000 .

In summary, the simulated results show that the uncontrolled system (6), that is, system (1), has simple dynamical behaviors, and the controlled system (7), that is, system (3) with controller (4), has complex dynamical behaviors; see Figures 1, 2, 3, and 4. The graphs presented indeed display an expected well-behaved structure in one moment, and after the chaotification treatment the referred system presents some behavior that resembles chaos. Nothing is less than expected. 


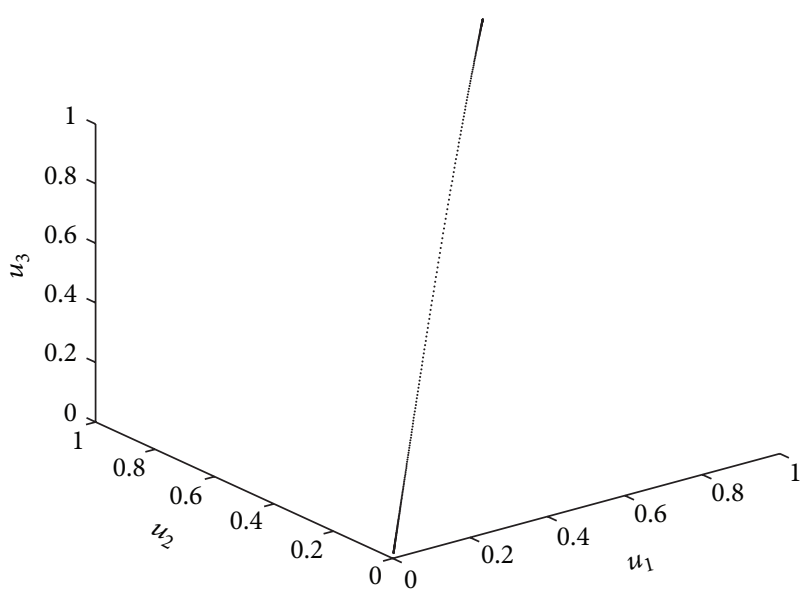

FIGURE 3: 3D computer simulation result shows simple dynamical behaviors of the uncontrolled system $(6)$ in the $\left(u_{1}, u_{2}, u_{3}\right)$ space for $k=2$, and $n$ from 0 to 20000, with the initial condition $u(0)=$ $(0.01,0.01,0.01)^{T}$.

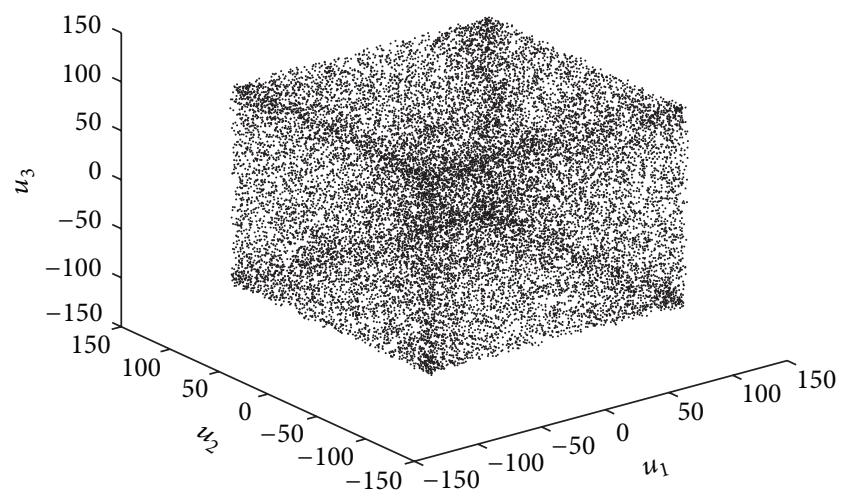

FigURE 4: 3D computer simulation result shows complex dynamical behaviors of the controlled system (7) in the $\left(u_{1}, u_{2}, u_{3}\right)$ space for $\alpha=$ $100, \beta=100 \pi, k=2$, and $n$ from 0 to 20000 , with the initial condition $u(0)=(0.01,0.01,0.01)^{T}$.

\section{Conclusion}

In this paper, we consider anticontrol of chaos for a class of delay difference equations via the feedback control technique. Based on the result that a regular and nondegenerate heteroclinic cycle connecting repellers for maps implies chaos, we establish a chaotification theorem. The controlled system is proved to be chaotic in the sense of both Devaney and Li-Yorke. It is noted that there are many delay discrete dynamical systems which have more than two fixed points. As all or some of the fixed points satisfy assumptions in Theorem 10, which are not very strict conditions, we can choose two of them and use the chaotification scheme established in this paper to chaotify these systems. Numerical simulations confirm the theoretical analysis. However, the map of the original system needs to satisfy some conditions in Theorem 10. Therefore, it is very interesting to explore the chaotification scheme for more general maps, which will be our further research.

\section{Conflict of Interests}

The author declares that there is no conflict of interests regarding the publication of this paper.

\section{Acknowledgments}

This research was supported by the National Natural Science Foundation of China (Grant nos. 11101246 and 11101247). The author would like to thank the editor and the anonymous referees for their valuable comments and suggestions, which have led to an improvement of this paper.

\section{References}

[1] W. J. Freeman, "Chaos in the brain: possible roles in biological intelligence," International Journal of Intelligent Systems, vol. 10, pp. 71-88, 1995.

[2] S. J. Schiff, K. Jerger, D. H. Duong, T. Chang, M. L. Spano, and W. L. Ditto, "Controlling chaos in the brain," Nature, vol. 370, pp. 615-620, 1994.

[3] M. E. Brandt and G. R. Chen, "Bifurcation control of two nonlinear of models of cardiac activity," IEEE Transactions on Circuits and Systems I, vol. 44, pp. 1031-1034, 1997.

[4] W. L. Ditto, M. L. Spano, J. Nelf et al., "Control of human atrial fibrillation," International Journal of Bifurcation and Chaos, vol. 10, pp. 593-602, 2000.

[5] G. Jakimoski and L. G. Kocarev, "Chaos and cryptography: block encryption ciphers based on chaotic maps," IEEE Transactions on Circuits and Systems. I. Fundamental Theory and Applications, vol. 48, no. 2, pp. 163-169, 2001.

[6] L. G. Kocarev, M. Maggio, M. Ogorzalek, L. Pecora, and K. Yao, "Special issue on applications of chaos in modern communication systems," IEEE Transactions on Circuits and Systems I, vol. 48, pp. 1385-1527, 2001.

[7] G. R. Chen and D. J. Lai, "Feedback control of Lyapunov exponents for discrete-time dynamical systems," International Journal of Intelligent Systems, vol. 6, pp. 1341-1349, 1996.

[8] G. R. Chen and D. J. Lai, "Anticontrol of chaos via feedback," in Proceedings of the IEEE Conference on Decision and Control, pp. 367-372, San Diego, Calif, USA, 1997.

[9] G. R. Chen and D. J. Lai, "Feedback anticontrol of discrete chaos," International Journal of Bifurcation and Chaos in Applied Sciences and Engineering, vol. 8, no. 7, pp. 1585-1590, 1998.

[10] G. R. Chen and Y. M. Shi, "Introduction to anti-control of discrete chaos: theory and applications," Philosophical Transactions of the Royal Society of London A. Mathematical, Physical and Engineering Sciences, vol. 364, no. 1846, pp. 2433-2447, 2006.

[11] X. F. Wang and G. R. Chen, "Chaotification via arbitrarily small feedback controls: theory, method, and applications," International Journal of Bifurcation and Chaos in Applied Sciences and Engineering, vol. 10, no. 3, pp. 549-570, 2000.

[12] Q. Chai, R. Loxton, K. L. Teo, and C. Yang, "A class of optimal state-delay control problems," Nonlinear Analysis. Real World Applications, vol. 14, no. 3, pp. 1536-1550, 2013.

[13] J. D. Zhu and Y. P. Tian, "Necessary and sufficient conditions for stabilizability of discretetime systems via delayed feedback control," Physics Letters A, vol. 343, pp. 95-107, 2005. 
[14] X. F. Wang and G. R. Chen, "Chaotifying a stable map via smooth-amplitude high-frequency feedback control," International Journal of Circuit Theory and Applications, vol. 28, pp. 305-312, 2000.

[15] Y. M. Shi, P. Yu, and G. R. Chen, "Chaotification of discrete dynamical systems in Banach spaces," International Journal of Bifurcation and Chaos in Applied Sciences and Engineering, vol. 16, no. 9, pp. 2615-2636, 2006.

[16] Z. C. Li, "Chaotification for linear delay difference equations," Advances in Difference Equations, vol. 2013, article 59, 2013.

[17] Z. C. Li, Y. M. Shi, and W. Liang, "Discrete chaos induced by heteroclinic cycles connecting repellers in Banach spaces," Nonlinear Analysis. Theory, Methods \& Applications, vol. 72, no. 2, pp. 757-770, 2010.

[18] F. Giannakopoulos and A. Zapp, "Local and global Hopf bifurcation in a scalar delay differential equation," Journal of Mathematical Analysis and Applications, vol. 237, no. 2, pp. 425-450, 1999.

[19] M. H. Jiang, Y. Shen, J. G. Jian, and X. X. Liao, "Stability, bifurcation and a new chaos in the logistic differential equation with delay," Physics Letters A, vol. 350, no. 3-4, pp. 221-227, 2006.

[20] Z. M. He, X. Lai, and A. Y. Hou, "Stability and Neimark-Sacker bifurcation of numerical discretization of delay differential equations," Chaos, Solitons and Fractals, vol. 41, no. 4, pp. 20102017, 2009.

[21] Y. Huang and X. Zou, "Co-existence of chaos and stable periodic orbits in a simple discrete neural network," Journal of Nonlinear Science, vol. 15, no. 5, pp. 291-303, 2005.

[22] M. Peng, J. C. Yu, and X. J. Wang, "Complex dynamics in simple delayed two-parameterized models," Nonlinear Analysis. Real World Applications, vol. 13, no. 6, pp. 2530-2539, 2012.

[23] T. Y. Li and J. A. Yorke, "Period three implies chaos," The American Mathematical Monthly, vol. 82, no. 10, pp. 985-992, 1975.

[24] R. L. Devaney, An Introduction to Chaotic Dynamical Systems, Addison-Wesley Publishing Company, New York, NY, USA, 1987.

[25] M. Martelli, M. Dang, and T. Seph, "Defining chaos," Mathematics Magazine, vol. 71, no. 2, pp. 112-122, 1998.

[26] C. Robinson, Dynamical Systems: Stability, Symbolic Dynamics and Chaos, CRC Press, Boca Raton, Fla, USA, 1995.

[27] S. Wiggins, Global Bifurcations and Chaos, Springer, New York, NY, USA, 1988.

[28] J. Banks, J. Brooks, G. Cairns, G. Davis, and P. Stacey, "On Devaney's definition of chaos," The American Mathematical Monthly, vol. 99, no. 4, pp. 332-334, 1992.

[29] W. Huang and X. D. Ye, "Devaney's chaos or 2-scattering implies Li-Yorke’s chaos," Topology and its Applications, vol. 117, no. 3, pp. 259-272, 2002.

[30] Y. M. Shi and G. R. Chen, "Chaos of discrete dynamical systems in complete metric spaces," Chaos, Solitons and Fractals, vol. 22, no. 3, pp. 555-571, 2004.

[31] Y. M. Shi and G. R. Chen, "Discrete chaos in Banach spaces," Science in China A. Mathematics, vol. 48, no. 2, pp. 222-238, 2005, Chinese version: vol. 34, pp. 595-609, 2004. 


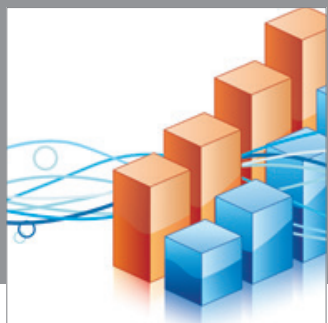

Advances in

Operations Research

mansans

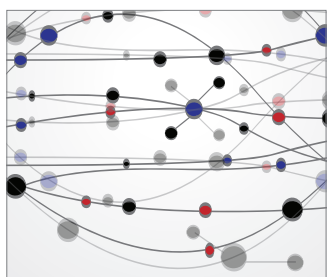

The Scientific World Journal
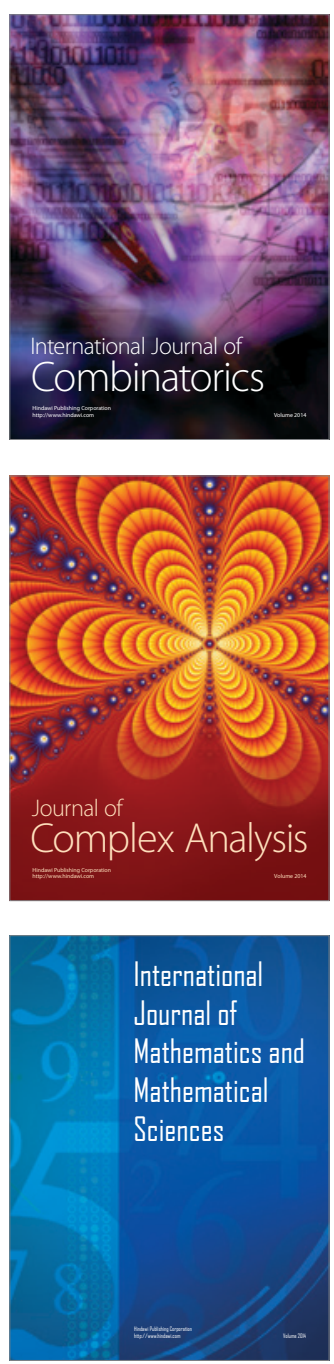
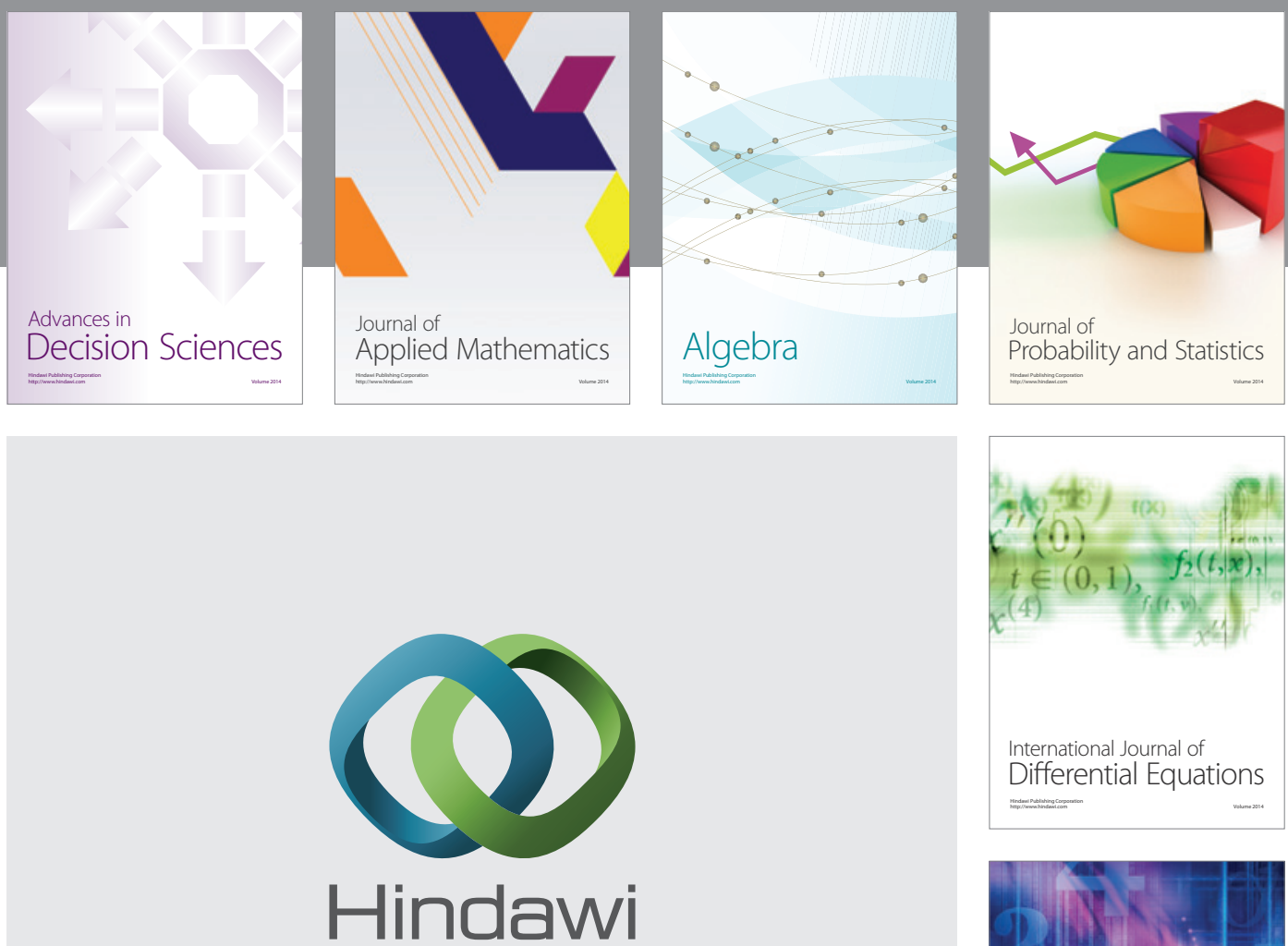

Submit your manuscripts at http://www.hindawi.com
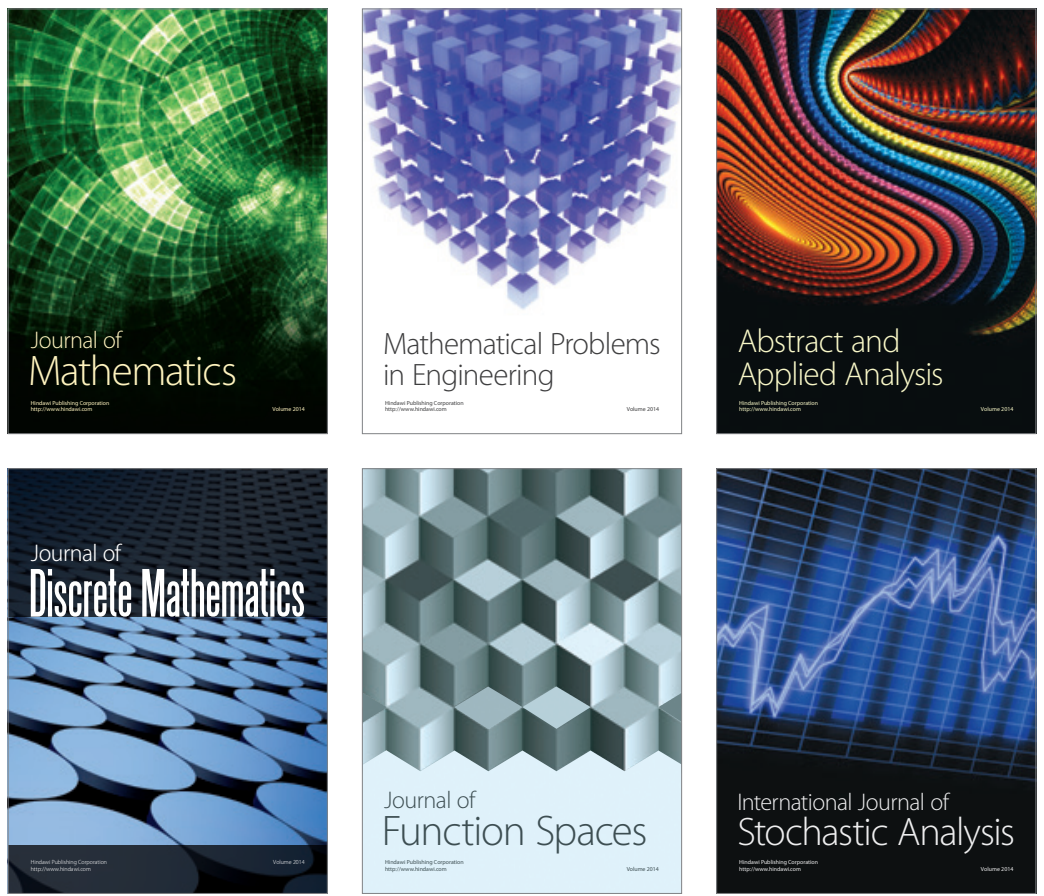

Journal of

Function Spaces

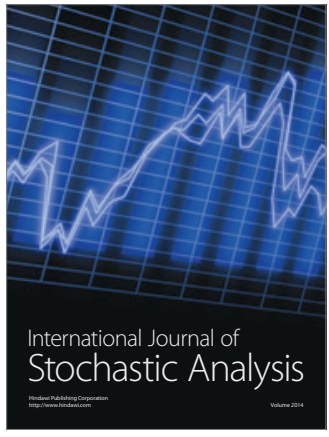

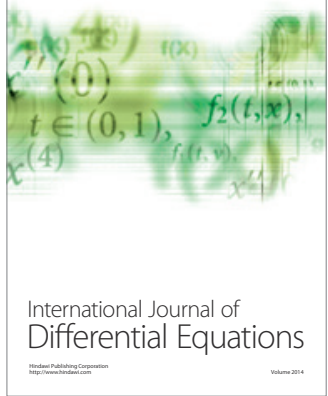
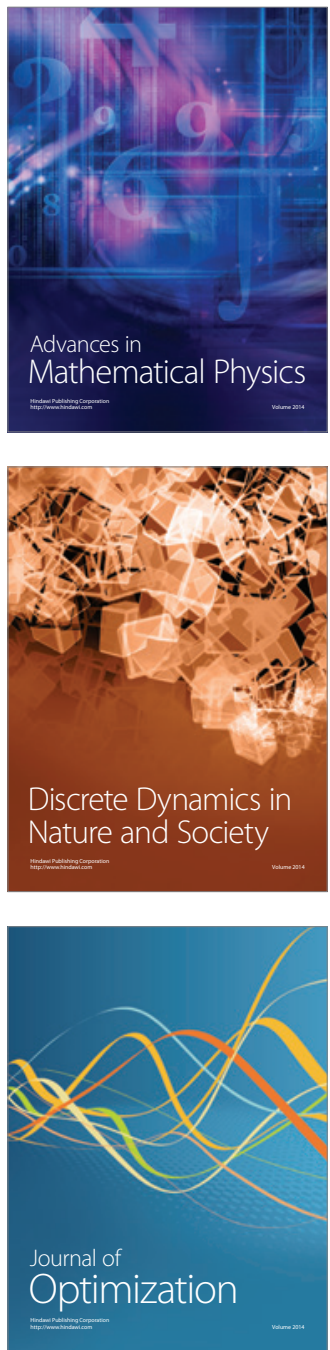